\title{
EFFECT OF DEFECT STRUCTURE ON LIGHT EXTRACTION FROM A PHOTONIC CRYSTAL SLAB NANOCAVITY
}

\author{
Jyh-Yang Wang, Yean-Woei Kiang, and C. C. Yang \\ Graduate Institute of Communication Engineering, Graduate Institute of Electro-Optical \\ Engineering, and Department of Electrical Engineering, National Taiwan University, \\ 1, Roosevelt Road, Section 4, Taipei, Taiwan \\ (phone) 886-2-23635251 (fax) 886-2-23652637 (e-mail) ywkiang@ntu.edu.tw
}

Abstract -- Radiation characteristics of photonic crystal slab nanocavities are numerically studied. The radiation rate and extraction efficiency are calculated for different defect structures.

In this paper, we numerically calculate the effective extraction rate for photonic crystal slab nanocavities with different defect structures and compare the differences among them. The structure we considered is shown schematically in Fig. 1. It is a dielectric slab standing in the air, penetrated with a $7 \times 7$ square lattice of air holes. The air hole at the center is specially processed (by varying the depth $d_{c}$ or radius $r_{c}$ ) to form a single defect as a nanocavity. The radius $r$ of other holes is fixed to be $0.35 a$ and the thickness $d$ of the slab to be $0.4 a$, where $a$ is the lattice constant. The refractive index of the slab is set to be 3.4. For simulations, the three-dimensional finite-difference time-domain (3-D FDTD) method is used. By discretizing in space and time with appropriate boundary conditions, the Maxwell's equations can be solved iteratively for whole computation domain at each time step. The excitation is chosen to be a y-directional oscillating dipole located at the center of the slab to excite the TE-like modes, simulating the light emission from a quantum well structure. An important measure of an LED is the extraction efficiency, which is defined here as the fraction of emitted flux through the top surface of the slab to the total emitted flux. We also calculate the radiation rate defined as the ratio of the total radiated power of a dipole in a nanocavity to the total radiated power of the same dipole in the free space. By multiplying the radiation rate and the extraction efficiency, a more practical quantity, the effective extraction rate, can be defined. After Fourier transforming the field evolution in time, we can compute all these derived quantities over a frequency range in a single simulation run. Figure 2 shows the effective extraction rate versus the normalized frequency $(a / \lambda)$ for the cavity without a central air hole $\left(d_{c}=r_{c}=0\right.$, denoted by the solid curve) and for the cavity with a central air hole $\left(d_{c}=0.1 a\right.$ and $r_{c}=0.35 a$, denoted by the dashed curve). It is found that the peak effective extraction rate is enhanced by about $33 \%$ at normalized frequency around 0.3 , if the central air hole is properly introduced.

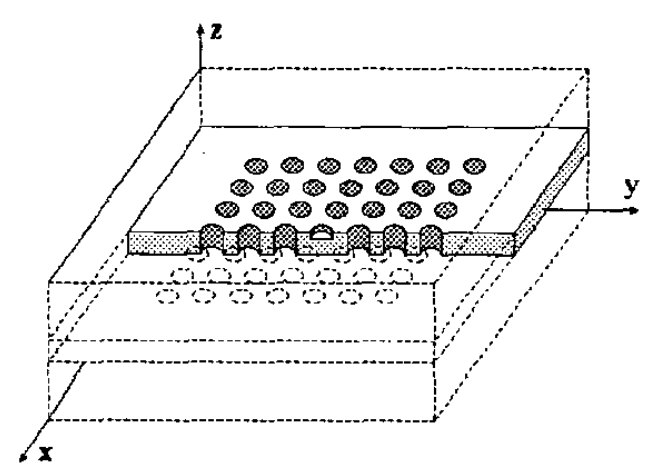

Fig. 1 Schematic diagram of a photonic crystal slab nanocavity (only half shown).

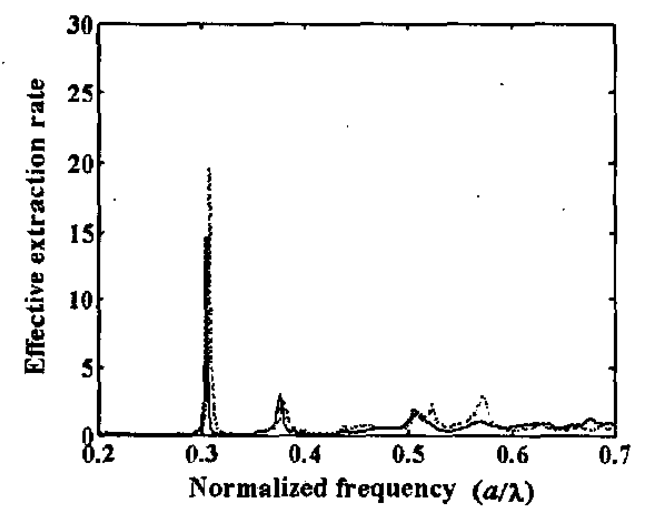

Fig. 2 The effective extraction rate versus the normalized frequency. The solid curve is for the cavity without a central air hole $\left(d_{c}=r_{c}=0\right)$ and the dashed curve for the cavity with a central air hole $\left(d_{c}=0.1 a\right.$ and $\left.r_{c}=0.35 a\right)$. 